\title{
Robust Adaptive Video Watermarking Algorithm based on Dual Transform Domains
}

\author{
De $\mathrm{Li}^{1}$, DaYou Jiang ${ }^{1}$ and JongWeon $\mathrm{Kim}^{2}$ \\ ${ }^{1}$ Department of Computer Science, Yanbian University, 133002, Yanji, China \\ ${ }^{2}$ Department of Intellectual Property, Sangmyung University \\ 110743, Seoul, Korea \\ leader1223@ybu.edu.cn,ybdxgxy13529@163.com,jwkim@smu.ac.kr \\ *Corresponding author: JongWeon Kim
}

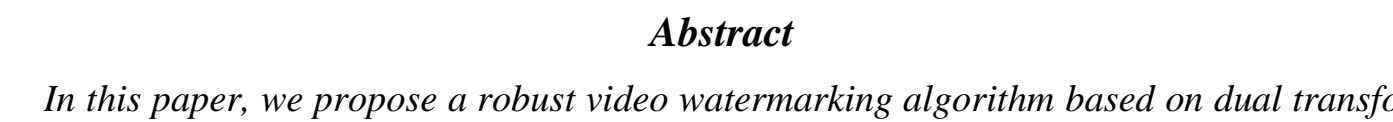

In thin paper, we propose a robust video watermarking algoth watermark, Particle Swarm Optimization algorth is used for embedding watermarks.

Firstly, one-level wavelet decomposition is employed for the two-dimensional carrier signal by using the characteristics of the cwavelet multi-gesolution analysis, and then the lowfrequency and middle-frequency coeffieients are extracted. After dividing the coefficients into small blocks, discrete cosine transforn is applied to each block coefficient.

Then, the adaptive quantization algorithm is designed. The optimal embedding positions in the two dimensional space of the carrier signahare determined dynamically by using Particle Swarm Optimization algorithm, and the coefficients in the optimal embedding positions are also extracted. According to the characteristics of these coefficients, the optimal quantization step sizes are determined dynamically in three dimensional space.

Finally, the watexmark is embedded into watermarking vector adaptively by using the optimal embedding positions and optimal quantization step sizes, then the watermarked vector will be received.

The experimental resuls show that compared with traditional watermark algorithms, the presented robust wafermark algorithm not only eliminate the block effects that might arise in the transform process, but also make full use of Particle Swarm Optimization algorithm to determine the loptimal embedding positions and optimal quantization step sizes of the watermark embedding algorithm. It has implemented the adaptive embedding and increased the non-sentience and robustness of the watermark. A series of attacks and comparative experiments show that the performance of the proposed algorithm is better than traditional algorthms.

Keywords: robust watermarking; dual-transform domain; particle swarm optimization algorithm; optimal embedding positions; optimal quantization step sizes

\section{Introduction}

Digital watermarking technique is an effective method to solve the copyright protection problems of digital media. At present, domestic and foreign research of digital watermarking algorithm involves a variety of digital carriers, the research of image watermarking is abundant, but the research of video watermarking is relatively less abundant. With the 
emergence of a large number of video products, video copyright protection has become an urgent demand.

First, Hartung, et al., [1] proposed a kind of additive watermark embedding algorithm in uncompressed video, that is $\mathrm{H} \& \mathrm{G}$ algorithm. In the watermark embedding algorithm, the original video signal is regarded as a one-dimensional signal. The process of this algorithm is a generalization of spread spectrum watermarking algorithm for static image [2]. Lancini, et al., [3] also proposed a spatial domain video watermarking algorithm based on human visual system. For the watermark embedding and extraction in the space domain is order to reduce the algorithm complexity. As processor performance continues to improve, the contradiction between large amounts of calculation is resolved in the watermark algorithm. The atermark embedding and extraction algorithm in the transform domain has been the most common algorithm. Shan, et al., [4] proposed a kind of real-time video watermarking algorithm based on Hadamar transform. Deguillaume, et al., [5] selected the mid-frequency part in threedimensional DFT coefficients of video sequence to embed watermark information. Swanson, et al., [6] proposed a watermarking algorithm that regards the video sequence as three dimensional signal, using three dimensional wavelettransform to process signal. Through the study of characteristics of MPEG-4 content based coding, Piva, et al., [7] proposed an algorithm to embed the watermark object in the MPEG-4 video stream. For video watermarking, select the transform domain to embed the owatermark, can comprehensive utilization of human visual characteristics and the imerent spatial and temporal characteristics of video data, to further inpprove the robustness of the watermarking system.

In this paper, we propose a dual transform domain watermarking algorithm based on 2D discrete wavelet transform (DWT) and 3D discrete cosine transform (DCT), combined with the adaptive Particle Swarm Optintization algorithm, in order to improve the robustness of video watermarking. The restof this paper is organized as follows. In Section 2, we introduce the principle of the adaptive quantization watermarking algorithm in dual transform domain and its specific process. Section 3 shows the simulation experiments with various attacks, and the test results are analyzed, compared to other similar algorithms with the same field, and verifies the robastness of the proposed watermarking system. Section 4 concludes this paper.

\section{Dual Transform Domain Adaptive Watermarking Algorithm}

\subsection{Particle Swarm Optimization algorithm}

Particle Swarm Optimization (PSO) algorithm is an Evolutionary Computation Technology proposed by Dr. Eberhart and Dr. Kennedy in 1995 [8]. Originally from the study of predatory behavior of birds, PSO algorithm is a simulation of birds of prey behavior. In the PSO algorithm, each solution of optimization problem is a bird within the search region, and it can be called a particle. All the particles have an initialization value and fitness value which is decided by the performance function, and each particle has a speed to determine the direction and distance it fly. All particles find the optimal solution through iteration. In every iteration, particles update their own position by tracking two extremums. One is the individual extremum pBest, particle itself to search, another one is the global extremum gBest, all current particles to search.

When the pBest and gBest are found, each particle will adjust its flight speed and the new location according to equation (1) and (2).

$$
\begin{gathered}
v^{\prime}=w^{*} v+c 1 * r 1 *(\text { pBest }- \text { present })+c 2 * r 2 *(\text { gBest }- \text { present }) \\
\text { present }{ }^{\prime}=\text { present }+v^{\prime}
\end{gathered}
$$


Among them, $v$ represents the initial velocity of a particle, $v$ ' represents the updated velocity of the particle, present represents the initial position of the particle, present' represents the updated new position of the particle. $w$ represents the inertia weight, it controls the particles velocity in each generation to update how much previous speed. c1, c2 as the acceleration factors, are used to control the particle impact of its optimal location and the global optimal location, and adjust the maximum step size of flight to pBest and gBest direction. $\mathrm{r} 1, \mathrm{r} 2$ are a random number betreen 0 and 1. In the Particle Swarm Optimization algorithm, inertia weight $w$, maximum speed $V \max$, and acceleration constant $\mathrm{c} 1, \mathrm{c} 2$, these parameters are set based on experience value.

The PSO algorithm in the actual execution is an iterative process to determine the optimal value. Its termination conditions need to be met: one is to reach the set updating maximum number of iterations; another one is to find the optimal value of the adjacency turotimes less than the set of the minimum values.

\subsection{Watermark Embedding Process}

The watermark embedding procedure is listed as follow:

Step1: Choose the I frames of the raw video sequence and separate them into groups, and each group consists of $\mathrm{N}$ frames.

Step2: Take 2D DWT for every frame of every group.

Step3: Divide the LH and HL coefficients of every frame of every group into $8 \times 8$ blocks.

Step4: Take 3D DCT for every block and obtain the 3D DC coefficients.

Step5: After the quantization embed the watermark into each 3D DC coefficients;

Step6: Perform the corresponding inverse transform, and reconstruct the video sequence, then save the vided

The principle of $2 \mathrm{D}$ DWT and $3 \mathrm{DPCT}$ is shown in Figure 1.

\subsection{Watermark extraction process}

The watermark extraction process is actually the inverse process of the watermark embedding. The extraction steps are extensively described below.

Step1: Choose the I frames of the raw video sequence and separate them into groups, and each-group consists of $\mathrm{N}$ frames.

Step2: T $\mathrm{ke}$ 2D DWT for every frame of every group.

Step3:Divide the LH and HL coefficients of every frame of every group into $8 \times 8$ blocks.

Step4: Take 3D DCT for every block and obtain the 3D DC coefficients.

Step5: According to the quantization algorithm, extract the embedded watermark.

The watermark extraction process is shown in Figure 2.

In the embedding algorithm, we fully consider the video date characteristics of twodimensional space, and apply the dual transform domain processing method which combines the advantages of two kinds of transformation. 


\subsection{Adaptive quantization algorithm}

In this paper, according to the characteristics of watermark carrier and the size of watermark, we make full use of the characteristics of 2D and 3D space as well as the characteristics of low and intermediate frequency part of the carrier, and apply the adaptive Particle Swarm Optimization algorithm to determine the optimal embedding position and the optimal quantization step.

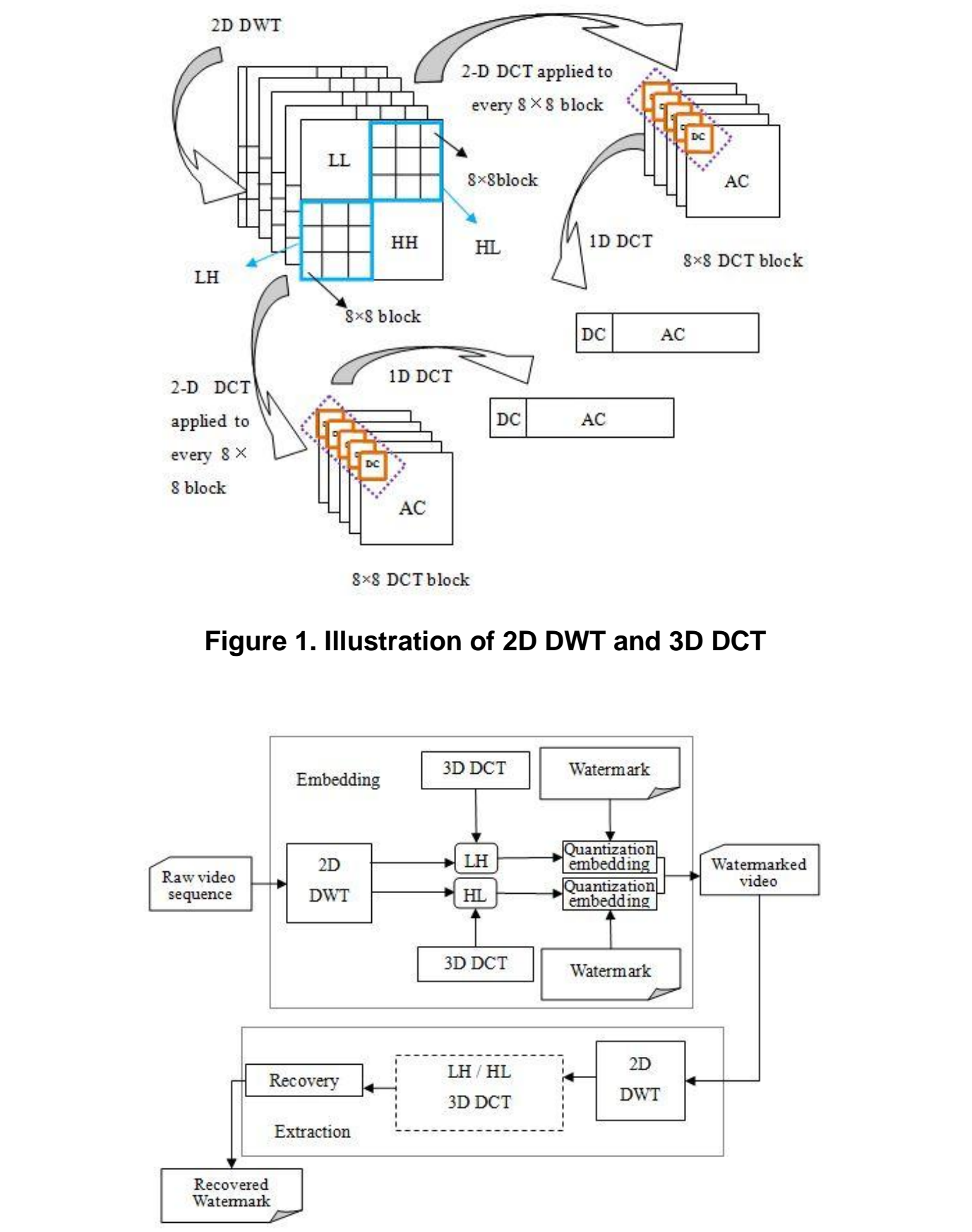

Figure 2. Process of the improved algorithm 


\subsubsection{To determine the optimal location of embedded:}

In the adaptive quantization algorithm proposed in this paper, we use PSO search approach to dynamically determine the optimal embedding location of the watermark in twodimensional space. The steps are extensively described below.

Step1: Choose the I frames of the raw video sequence and separate them into groups, and each group consists of $\mathrm{N}$ frames.

Step2: Apply 2D DWT to every frame of every group.

Step3: Divide LL into $8 \times 8$ blocks.

Step4: Apply the first DCT to each $8 \times 8$ block, and get the DC coefficients and AC coefficients.

Step5: Specify the same quantization step according to the experience yalue for all the small pieces of the LL coefficients part, and use the PSO algorithm for searching, then train it by low-pass filter attack.

Step6: Evaluate the performance of the PSO algorithm according to the peak signal-to-noise ratio (PSNR) and Normalized correlation (NC), and save the optimal embedding position of the LL coefficients part.

The same process is used for the horizontal coefficient (HL) of 2D DWT, namely the intermediate frequency part. The difference is that we choose the Gauss noise attack and salt \& pepper noise attack to train the PSO algorithm at this time. Similarly, we save the best embedding position of HL coeffrcients part when the performance evaluation function obtains the optimal value.

The process of the algorithm is described as shown in Figure 3.

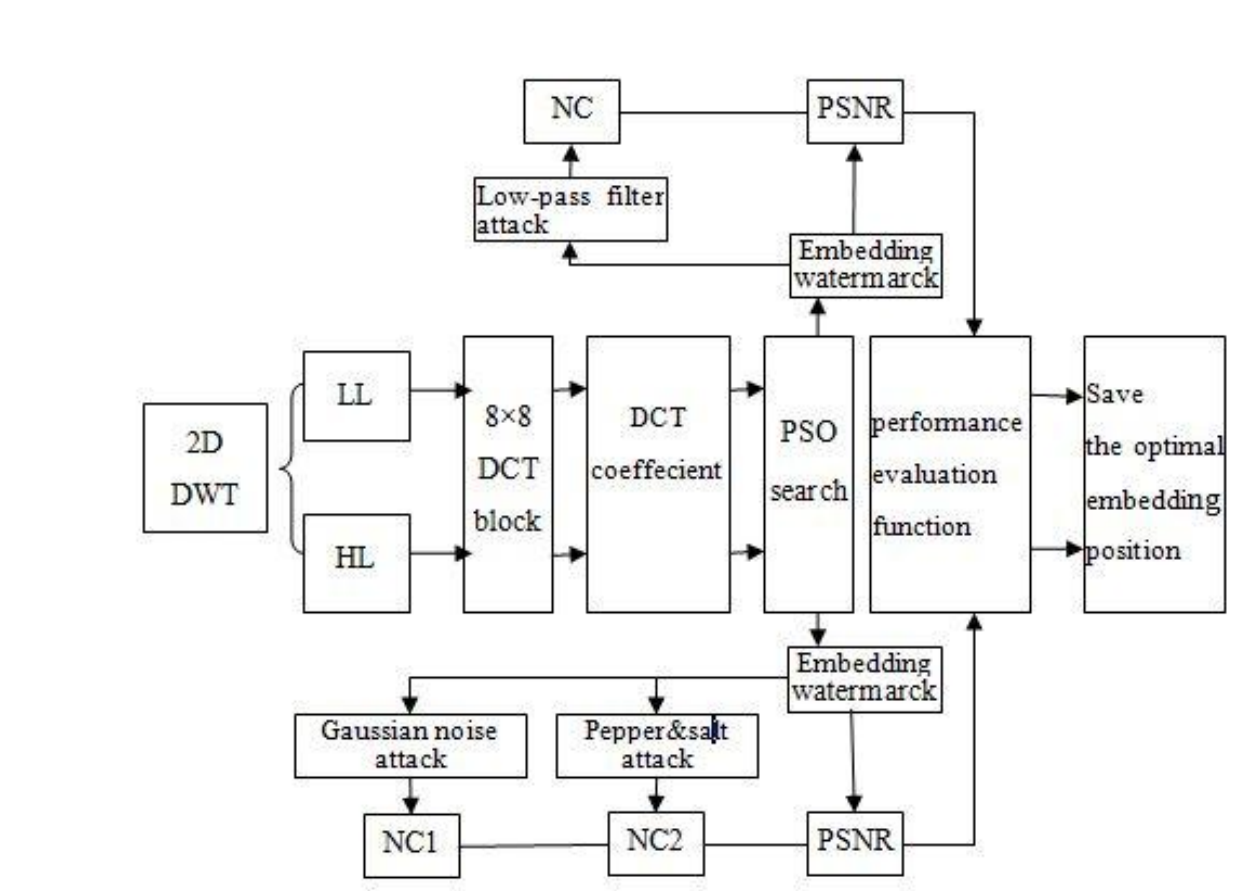

Figure 3. Search process of the optimal embedding position 


\subsubsection{To determine the optimal quantization step:}

The optimal quantization step determines the imperceptibility and anti-attack capability of the watermark. The determination of the optimal quantization step is based on the determination of the optimal embedding position. The specific process is described as follows:

Step1: Remove the optimal embedding position coefficients of every $8 \times 8$ block that are at the same time axial position in the each group of frames, the three-dimensional vector is composed of these coefficients.

Step2: Apply 1D DCT to each vector in the time axial direction from vector group,and obtain the DC coefficients matrix after performing 3D DCT.

Step3: Embed the watermark into the obtained DC coefricients matrix And use PSO algorithm for searching, then take the low-pass fitrer attack to train the low frequency part, and also take the Gauss noise and salt-pepper nojse attack to train the intermediate frequency part.

Step4: Evaluate the performance of the PSO algorithm aocording to the PSNR and NC, and save the optimal quantization step of the low coefficients part and intermediate frequency part individually.

\section{Experimental Results and Analysis}

We have performed various experiments in order to test the performance of the proposed adaptive video watermarking algorithm based on dual transform domain. Through the analysis of the expentment results, we yerify the effectiveness of the algorithm.

In the experments, we used the ancompressed video with size $320 \times 240$ and it consists of 100 frames. The watermark image is denoted by a binary image, the size of which is $20 \times 15$, as shown in Figure 4.
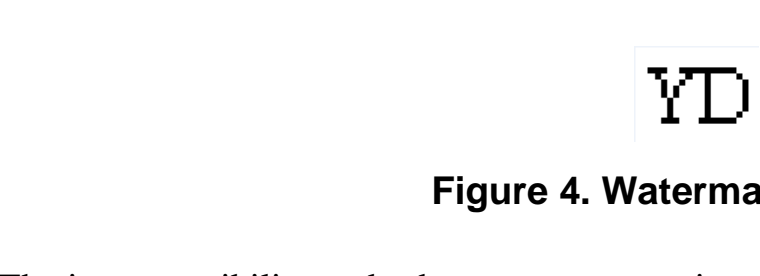

\section{Figure 4. Watermark image}

The imperceptibility and robustness are very important factors to measure the performance of the watermarking system. The performance evaluation function is defined as follows.

$$
\text { fitness }=P S N R * \prod_{i=1}^{m} N C_{i}
$$

Among them, the peak signal-to-noise ratio (PSNR) is used to measure the imperceptibility of watermarking, and normalized correlation (NC) is used to measure the robustness of the watermarking. $m$ stands for the numbers of different attack types. In this paper, this function also used to evaluate the global optimal value in PSO algorithm. 
To verify the effectiveness of the algorithm, we selected multiple video files for the experiments. As shown in Table 1, in the selection of experimental carriers, there is a No.2 video file that contains abundant high frequency information, a No.3 video file that contains abundant low frequency information, and also No.1 and No.4 video files that contain homogeneous distribution frequency information. Without any attack situations, all kinds of video watermark embedding and extraction results are shown in Table 1.

Table 1. Effect diagram of watermark embedding and extraction

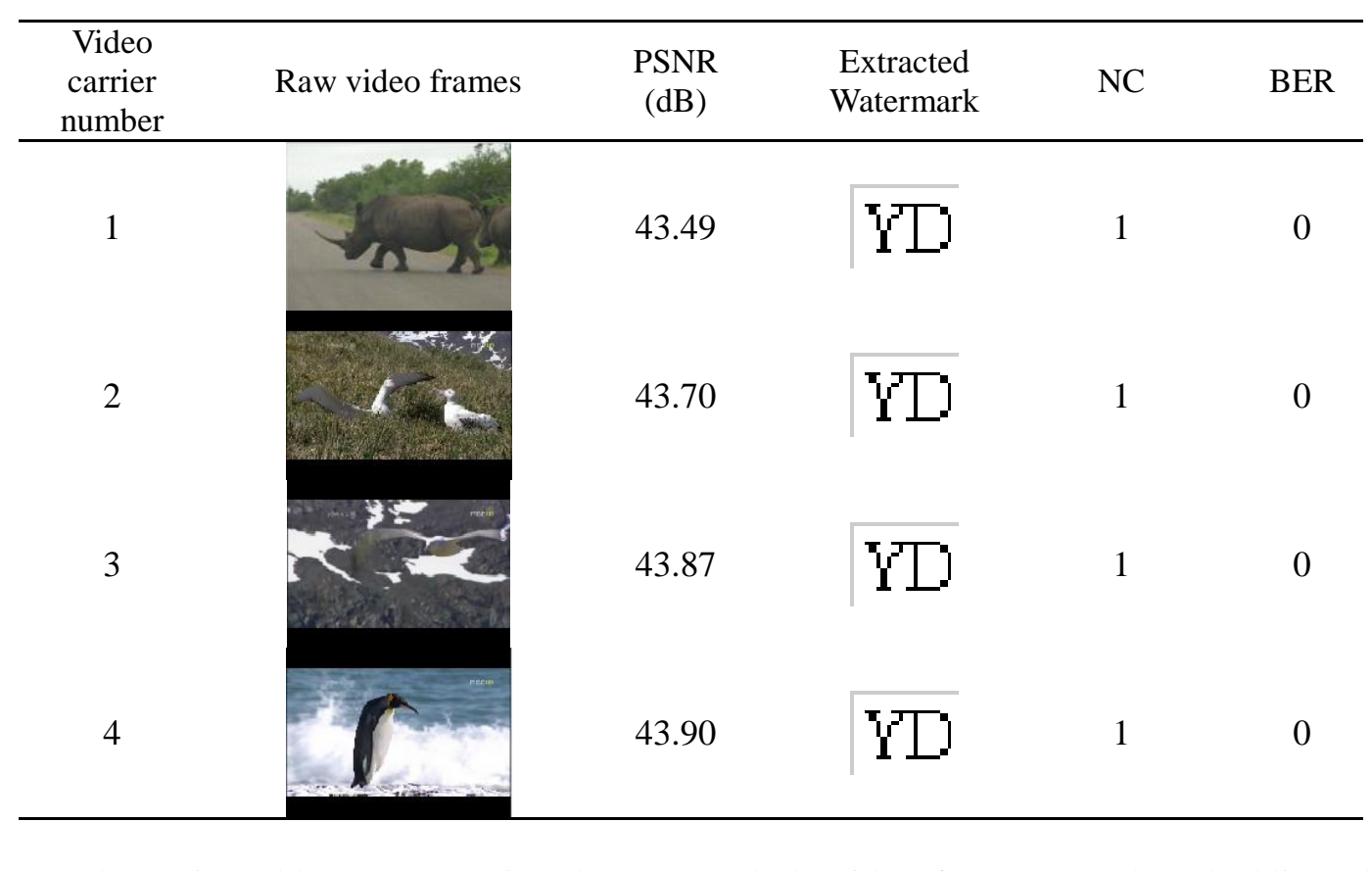

As shown in Table 1, when using the proposed algorithm for watermark embedding, the PSNR values of different types of video files are more than $43 \mathrm{~dB}$, and the difference between the watermarked video and the cover video is unperceivable. In addition, the similarity of the extracted watermar $\mathbf{K}$ and original watermark $(\mathrm{NC})$ is 1 ; the bit error rate (BER) is 0 . It is evident that the proposed algorithm can accurately extract the watermark without error.

\subsection{The attack experiment}

L order to test and verify the performance of the algorithm, in this paper, we selected the high frequency information and the low frequency information are distributed uniformly of No.1 video files as the experimental object. We conducted a series of experiments including the Gauss noise and pepper \& salt noise (shown in Table 2), low pass filter (shown in Table 3), rotation (shown in Table 4), frame drop, frame exchange (shown in Table 5), and MPEG-4 compression (shown in Table 6) attacks. In the frame exchange experiment, for the first time we chose two frames from the same group, and exchanged the first frame and the ninth frame images; for the second time we chose two frames from different groups, and exchanged the fifth frame and the twenty-first frame images. 
Table 2. Extracted results of noise attacks

\begin{tabular}{|c|c|c|c|c|}
\hline & variance & 0.0005 & 0.003 & 0.0055 \\
\hline \multirow{5}{*}{ Gaussin } & $\begin{array}{l}\text { Attacked } \\
\text { Frame }\end{array}$ & & & \\
\hline & $\begin{array}{c}\text { Extracted } \\
\text { Watermark }\end{array}$ & & & \\
\hline & $\mathrm{NC}$ & 1 & 1 & \\
\hline & BER & 0 & 0 & \\
\hline & $\begin{array}{c}\text { Noise } \\
\text { Density }\end{array}$ & 0.002 & 0.025 & \\
\hline Pepper & $\begin{array}{l}\text { Attacked } \\
\text { Frame }\end{array}$ & & & \\
\hline salt & $\begin{array}{l}\text { Extracted } \\
\text { Watermark }\end{array}$ & & & \\
\hline & $\mathrm{NC}$ & & & 0.92 \\
\hline & BER & & 0.02 & 0.07 \\
\hline
\end{tabular}

Table 3. Extracted results of low-pass filter attacks

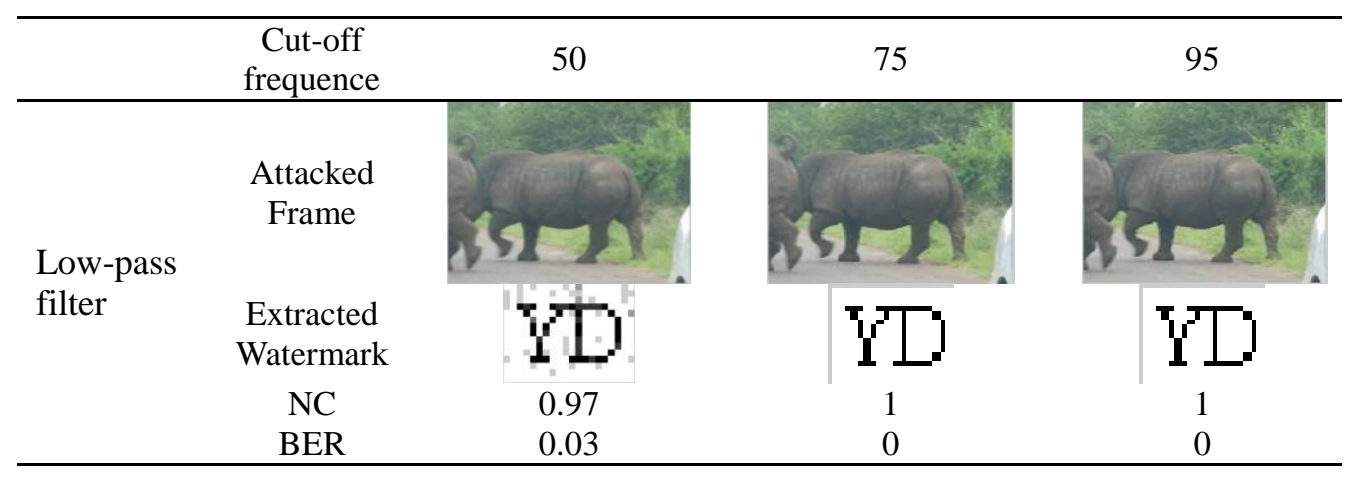

Table 4. Extracted results of rotation attacks

\begin{tabular}{|c|c|c|c|c|}
\hline & $\begin{array}{c}\text { Angle of } \\
\text { rotation }\end{array}$ & $10^{\circ}$ & $45^{\circ}$ & $60^{\circ}$ \\
\hline \multirow{3}{*}{ Rotation } & $\begin{array}{l}\text { Attacked } \\
\text { Frame }\end{array}$ & & & \\
\hline & $\begin{array}{c}\text { Extracted } \\
\text { Watermark }\end{array}$ & & & \\
\hline & $\begin{array}{c}\mathrm{NC} \\
\mathrm{BER}\end{array}$ & $\begin{array}{l}0.93 \\
0.06\end{array}$ & 0.86 & $\begin{array}{l}0.79 \\
0.17\end{array}$ \\
\hline
\end{tabular}


Table 5. Extracted results of frame attacks

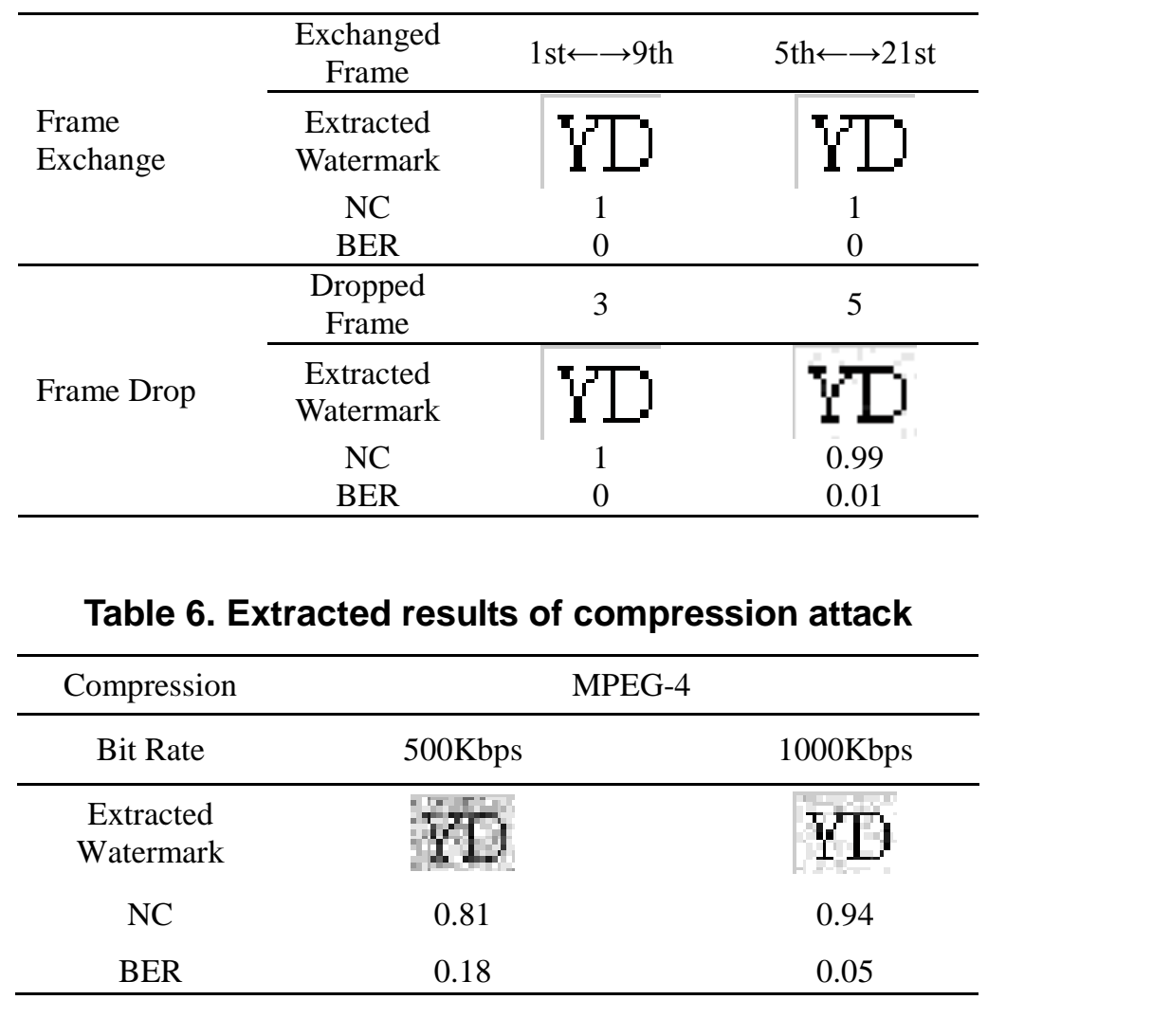

As shown in Table 2 to Table 6 , Even the video under different attacks, we still could extract the watermark information identifiable from it. It is proved that this algorithm has strong robustness.

\subsection{The comparative experiments}

In this paper, two groups of comparative experiments were selected. In the first group, we compared the dual transform domain watermarking algorithm with the original algorithm on the pros and cons of the performance. In the second group, we compared the adaptive quantization algorithm with the commonly used dithering quantization algorithm on the pros and cons of the performance. We ensured that the PSNR values are approximately equal after embeding the watermark, and compared the NC values of two algorithms. The experimental results for the first compared group are shown in Figure 5 to Figure 6. The experimental results for the second compared group are shown in Figure 8 to Figure 11.

Figure 5 and Figure 8 shows the result of the pepper \& salt attack, Figure 6 and Figure 9 shows the result of the Gauss noise attack, Figure 7 and Figure 10 shows the result of the frame drop attack, and Figure 11 shows the result of the low-pass filter attack. The NC value corresponding to the conventional algorithm is marked with the circle diagram ( $\bigcirc$ ), The NC value corresponding to the application algorithm in this paper is marked with an asterisk $(\times)$. As can be seen from the picture, both the watermark algorithms based on the dual transform 
domain and based on the adaptive quantization algorithm improve the robustness of the algorithm.
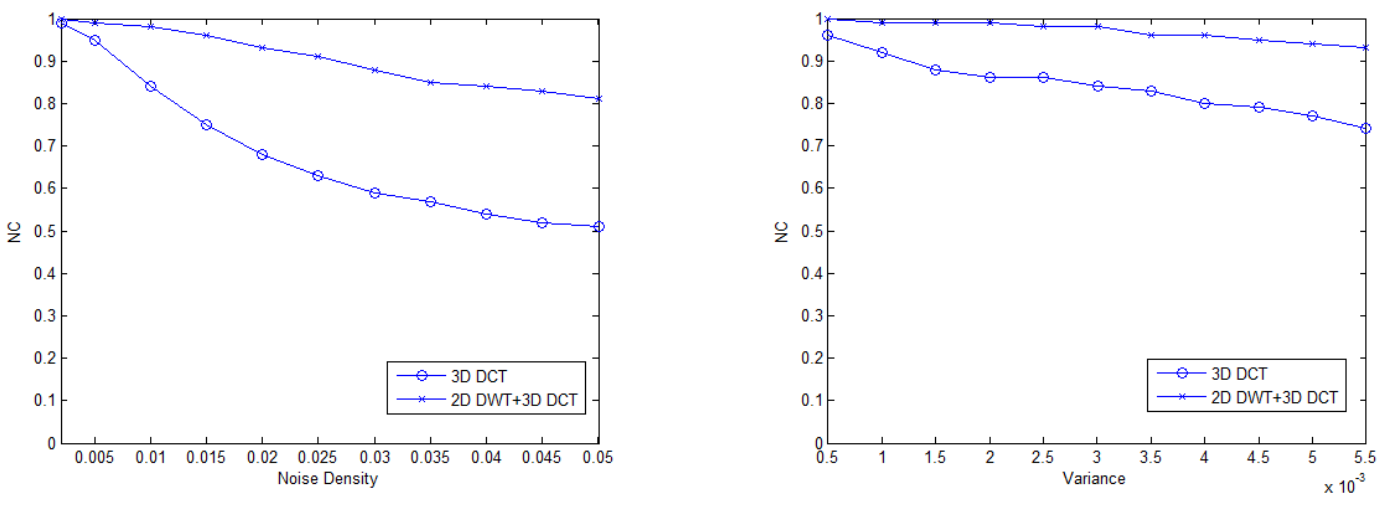

Figure 5. Salt \& Pepper attacks

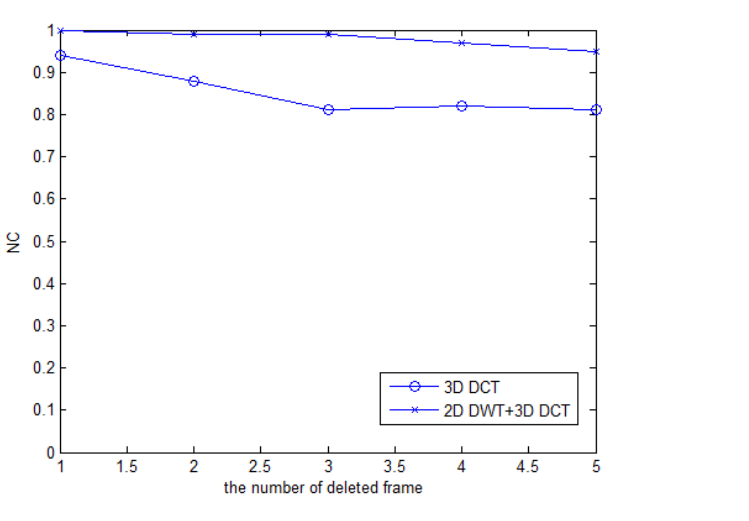

Figure 7. Frames drob attacks

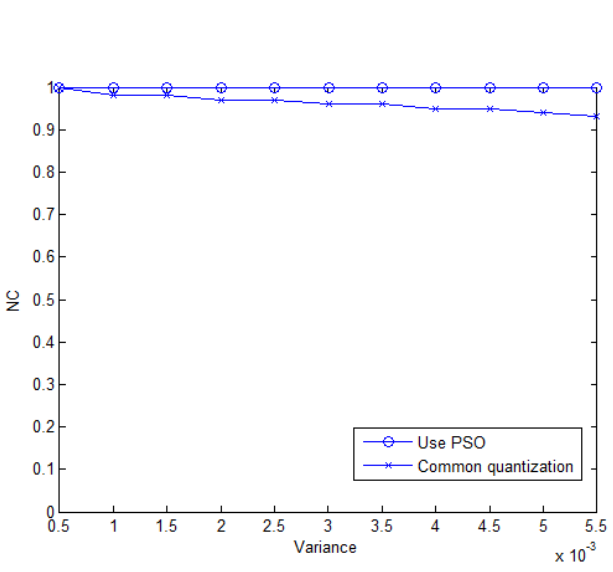

Figure 9. Gaussian attacks

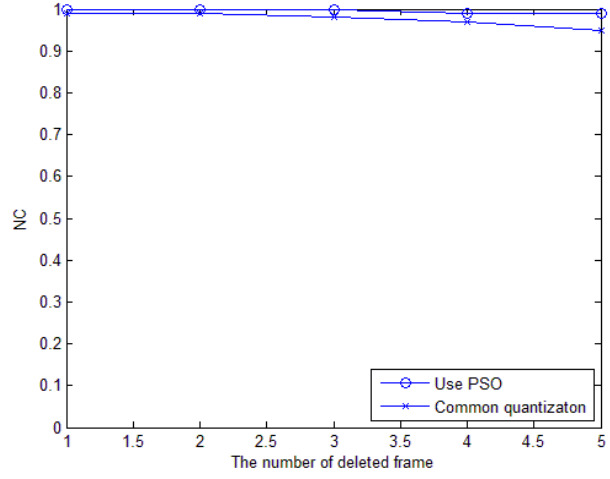

Figure 10. Frames drop attacks 


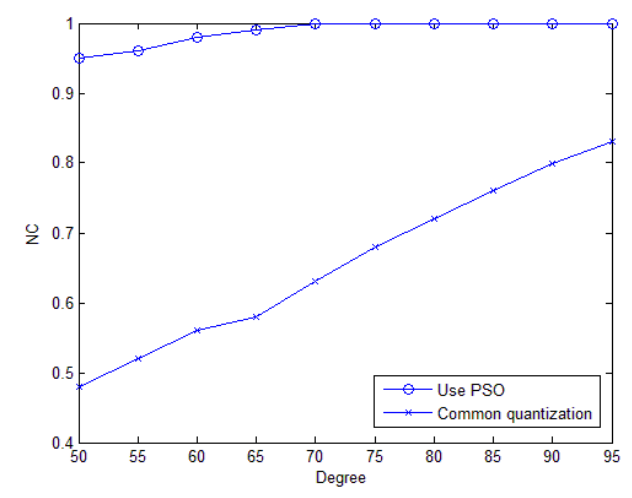

Figure 11. Low-pass filtering attacks

This paper also conducted a comparative analysis of attack experiments for other different types of video files; the results are similar to the above experimental results. The robustness for the trained attack types was improved quite obviously. For untrained attack types, the robustness is was also slightly increased. The above experimental results show that the proposed watermarking algorithm is effective and has strongrobustness.

\section{Conclusions}

This paper has proposed a robust digital watermarking algorithm, which is combined the 3D spatial watermarking algorithm based on the dual transform domain with the adaptive quantization watermarking algorithm based op Particle Swarm Optimization, and improved the imperceptibility and robusthess of the watermark. The dual transform domain algorithm fully combined the mullti-resolution analysis property of DWT with the shaped property of DCT. Particle Swarm Optimization algorithm based on common quantization algorithm and its own characteristics, combined wdth its property for solving global optimization, the watermark can be embedded adaptively. Experimental results demonstrate that the proposed algorithm has good imperceptibility and robustness against a variety of common video processing attacks including pepper \& salt noise, Gaussian white noise, low-pass filter, rotation, frame drop, frame exchange, and MPEG-4 compression.

\section{Acknowledgements}

This research project was supported by the Ministry of Culture, Sports and Tourism (MCST) and the Korea Copyright Commission in 2011.

\section{References}

[1] F. Hartung and B. Girod, "Watermarking of Uncompressed and Compressed Video", Signal Processing, Special Issue on Copyright Protection and Access Control for Multimedia Services, vol. 66, no. 3, (1998), pp. 283-301.

[2] I. J. Cox, J. Kilian, T. Leighton and T. Shamoon, "Secure Spread Spectrum Watermarking for Multimedia", IEEE Signal Processing Society, vol. 6, no. 12, (1997), pp. 1673-1687.

[3] R. Lancini, F. Mapelli and S. Tubaro, "A Robust Video Watermarking Technique in the Spatial Domain", Proceedings of International Symposium on Video/Image Processing and Multimedia Communications, Zadar, Croatia, (2002), pp. 251-256.

[4] A. Shan and E. Salari, "Real-time Digital Video Watermarking", Proceedings of International Conference on Consumer Electronics 2002, Los Angeles, USA, (2002), pp. 12-13. 
[5] F. Deguillarme, G. Csurka, J. O. Ruanaidh, et al., "Robust 3D DFT Video Watermarking", Proceedings of SPIE Security and Watermarking of Multimedia Contents, San Jose, USA, (1999), pp. 113-124.

[6] M. D. Swanson, B. Zhu, et al., "Multi-resolution Scene-based Video watermarking using perceptual models", IEEE Journal on Selected Areas in Communications, vol. 16, no. 4, (1998), pp. 540-550.

[7] A. Piva, R. Caldelli and A. D. Rosa, "A DWT-based Object Watermarking System for MPEG-4 Video Streams”, IEEE International Conference on Image Processing. Vancouver, British, vol. 3, (2000), pp. 5-8.

[8] M. Alfredo and S. Valentino, "Optimizing Web Content Presentation: A Online PSO Approach", 2009IEEE/WIC/ACM International Conference on Web Intelligence and Intelligent Agent Technology, Milan, Italy, vol. 3, (2009), pp. 26-29.

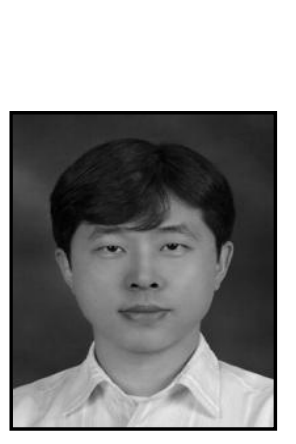

\section{Authors}

De Li received the Ph.D. degree from Sangmyung University, major in computer science in 2005. He is currently a professor of Dept. of Computer Science at Yanbian University in China, He is also a Principal Researcher at Copyright Protection Research Institute, Sangmyung University. His research interests are in the areas of copyright protection technology, digital watermarking, and digital forensic marking.

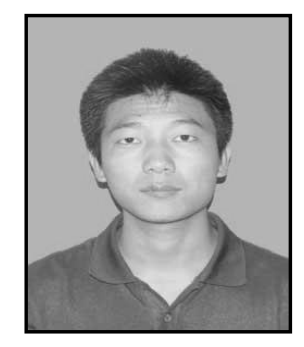

DaYou Jiang is a postgraduates major in Information Security, now studying at Yanbiah University in China. Her research interests are in the areas of copyright protection technology, information security, digital watermarking.
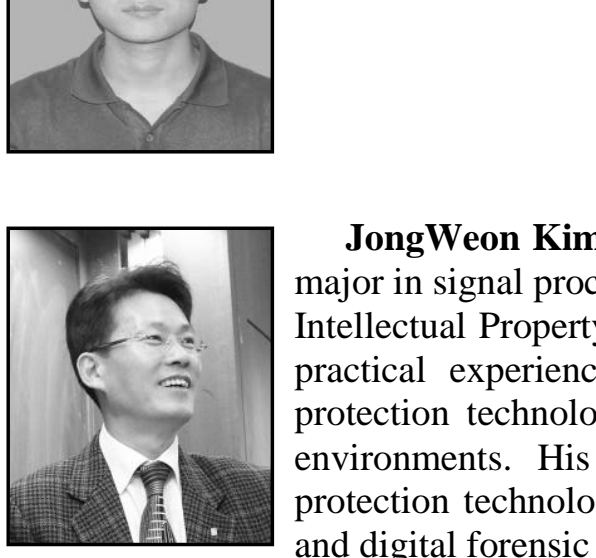

JongWeon Kim received the Ph.D. degree from University of Seoul, major in signal processing in 1995 . He is currently a professor of Dept. of Intellectual Property at Sangmyung University in Korea. He has a lot of pradtical experiences in the digital signal processing and copyright protection technology in the institutional, the industrial, and academic environments. His research interests are in the areas of copyright protection technology, digital rights management, digital watermarking, and digital forensic marking. 